\section{CONFERENCE WILL LOOK AT FUTURE OF DENTISTRY}

On 29 October 2014 Government Knowledge is holding an event called 'The future of dentistry and oral health in England' at Arlington Conference Centre in Camden, London.

From April 2013 the NHS Commissioning Board has been responsible for commissioning NHS dental services. Ensuring that dental services are equitable and accessible is essential in building good oral health, whether they are NHS or private dental services.

This informative policy-led event will take an in-depth look at the changes to NHS dental services as well as looking at the whole of the dental industry.

Key issues to be discussed include:

- Securing excellence in commissioning NHS dental services

- Creating high quality pathways for dentistry with a focus on outcomes

- Creating rewarding careers for clinicians

- Building public confidence in dental regulation

- Local Professional Networks

- The Dental Contract Pilots

- Innovations in dental treatment and technology

- Improving the oral health of children.

To find out more and to register for updates, visit www.govknow. com/briefing-detail.html?id=918\&t $\mathrm{nfo}=$ overview \&tsrc $=\mathrm{gk}$.

\section{IDEAL INTRODUCTORY LASER}

Quicklase have released the new 3 w laser that is ideal as an introductory laser and priced at only $£ 2,495$ including VAT and delivery. The $3 \mathrm{w}$ laser can be used for day to day dentistry for basic procedures such as troughing for accurate impressions and gingival re-contouring for better fitting veneers and crowns. It can also be used for gingivectomy, gingivoplasty and implant recovery.

The laser is also available as a $4 \mathrm{w}$ and $8 \mathrm{w}$ model as well as an $8 \mathrm{w}$ dual, which has an $810 \mathrm{~nm}$ and $910 \mathrm{~nm}$ wavelength.

In addition to soft tissue the Dentalase lasers can also be used for teeth whitening and TMJ, and come with therapy and whitening attachments at no extra cost.
Quicklase are the only British manufacturer of soft tissue lasers providing you with local backup and support. This also means that they can offer the Dentalase lasers at very competitive prices, making it affordable for your surgery to offer laser assisted dentistry and in turn making your practice more profitable.

Quicklase have also released endo products, which include K-Files, hand files, gutta percha, rotary files and paper point. Visit www.quicklase.com/product/endo/ for more information on endo products. www.quicklase.com

\title{
PROVIDE EFFECTIVE AFTERCARE TO YOUR PATIENTS
}

Tandex provide quality Orthodontic Kits to help aid your patients' aftercare following treatment.

An effective way to ensure that patients maintain efficient oral healthcare after a dental procedure is complete is to offer a fully prepared kit that contains all of the necessary tools.

The Tandex Orthodontic Kit has a selection of adjuncts ranging from an Advance Medium toothbrush to eliminate bacteria from around the oral cavity, to the SOLO Medium brush for precise interdental cleaning. It also comes with four FLEXI Lime interdental brushes with nonslip flexible grip for pleasant and versatile maintenance.

The kit includes a detailed user guide that comprehensively instructs patients on optimum cleaning protocols. Produced in cooperation with dentists and hygienists the in-depth information allows patients to use the products as they were intended, for easily achievable and excellent results.

Tandex Orthodontic Kits offer your patients the highest quality tools and direction for effective aftercare. Contact the team at Tandex today to find out more. www.tandex.dk

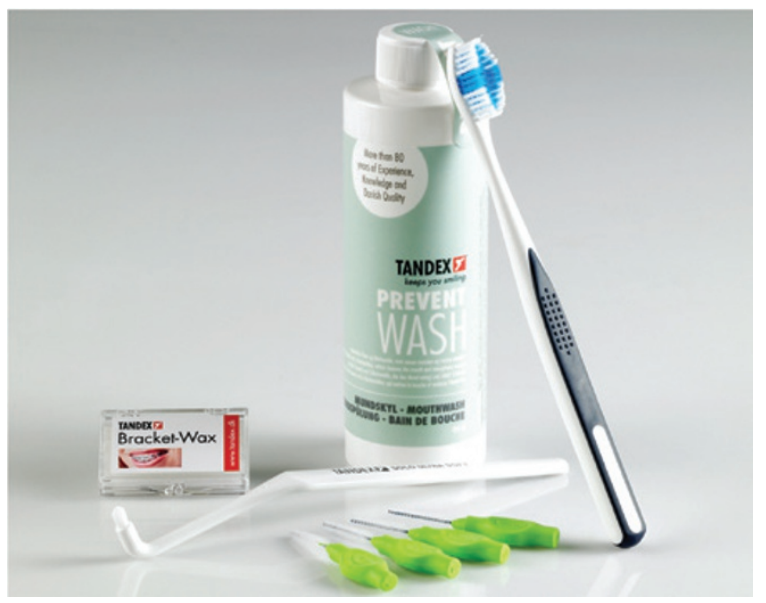

\section{CONNECT TO TRENDING TOPICS IN DENTISTRY}

GC has recently launched a fantastic new aid with 'GC get connected'. This newsletter is packed full of interesting and vital developments in the market place with clinical articles, step by step guides and tips to create a more successful practice for you.

Examples from the latest 'GC get connected' include:

- Fibres (un)limited (Dr Filip Keulemans)

- Tips and strategies for restoring large cavities using fibre-reinforced material (Drs Stephane Brouwet and Javier Tapia Guadix)

- Clinical efficiency of one-step self-etch adhesives versus etchand-rinse systems (Professor Jan van Dijken)

- Success with luting cements: material and technique tips (Dr Frédéric Raux)

- 10 years INITIAL: the birth and evolution of a highly innovative class (MDT Michael Brüsch)
- Step by step: Using EverX posterior.

'GC get connected' provides you an overview of the most innovative and trending topics in dentistry.

Simply log on to www. gceurope.com/news/newsletter and get connected now.

For further information contact GC UK on 01908218999. 